\section{Waning Antibody Responses in Asymptomatic and Symptomatic SARS-CoV-2 Infection}

\author{
Pyoeng Gyun Choe, ${ }^{1}$ Chang Kyung Kang, ${ }^{1}$ \\ Hyeon Jeong Suh, Jongtak Jung, \\ Kyoung-Ho Song, Ji Hwan Bang, Eu Suk Kim, \\ Hong Bin Kim, Sang Won Park, Nam Joong Kim, \\ Wan Beom Park, Myoung-don Oh
}

Author affiliation: Seoul National University College of Medicine, Seoul, South Korea

DOI: https://doi.org/10.3201/eid2701.203515

We investigated the kinetics of severe acute respiratory syndrome coronavirus 2 neutralizing antibodies in 7 asymptomatic persons and 11 patients with pneumonia. The geometric mean titer of neutralizing antibodies declined from 219.4 at 2 months to 143.7 at 5 months after infection, indicating a waning antibody response.

$\mathrm{N}$ eutralizing antibodies develop in asymptomatic persons with severe acute respiratory syndrome coronavirus 2 (SARS-CoV-2) infection; however, the initial immune response is not as strong as in patients with more severe disease $(1,2)$. We investigated the kinetics of SARS-CoV-2 neutralizing antibodies during the 5 months after infection in asymptomatic persons and patients with pneumonia caused by SARS-CoV-2.

We studied 7 persons infected with SARS-CoV-2 who were isolated in a community treatment center operated by Seoul National University (SNU) Hospital in Daegu, South Korea (3). Comprehensive monitoring confirmed that these 7 patients were asymptomatic (4). We also evaluated 11SARS-CoV-2positive patients with pneumonia at the Biocontainment Unit in the SNU Hospital and SNU Bundang Hospital. We classified each case of pneumonia as subtle (i.e., infiltrations observed only on computed tomography) or apparent (i.e., infiltrations observed on plain chest radiograph) (Appendix Table, https:/ / wwwnc.cdc.gov/EID/article/27/1/20-3515-App1. pdf). All patients provided informed consent.

We evaluated the antibody responses at 2 and 5 months after infection, as reported (1). We semiquantitatively measured IgG against SARS-CoV-2 using ELISA (Euroimmun, https:/ / www.euroimmun.com) with the recombinant S1 domain of the SARS-CoV-2 spike protein as the antigen. We interpreted the

${ }^{1}$ These first authors equally contributed to this article. optical density ratio (sample/calibrator) as negative $(<0.8)$, borderline $(\geq 0.8$ to $<1.1)$, or positive $(\geq 1.1)$, according to the manufacturer's recommendations. We also conducted neutralization assays as previously described (5) using BetaCoV/Korea/SNU01/2020 virus (6) and 2-fold serially diluted plasma samples (24,096-fold). We recorded the highest dilution of plasma that showed inhibition activity of SARS-CoV-2 as the neutralizing antibody titer. We considered a $\geq 4$-fold reduction in antibody titer to be a waning response. The Institutional Review Boards of Seoul National University Hospital approved the study (IRB no. H-2004-158-1118).

Two months after infection, $11(100 \%)$ patients with pneumonia and $5(71 \%)$ with asymptomatic infection had positive ELISA results. Five months after infection, $5(100.0 \%)$ patients with apparent pneumonia, $5(83.3 \%)$ with subtle pneumonia, and $4(57.1 \%)$ with asymptomatic infection had positive ELISA results. The mean ELISA optical density decreased significantly from 2 to 5 months after infection (4.93 at 2 months vs. 4.09 at 5 months; $p=0.01$ ).

Two months after infection, all patients had neutralizing antibodies. Antibody titers correlated with disease severity; the geometric mean titer was 105 among symptomatic persons, 161 among patients with subtle pneumonia, and 891 among patients with apparent pneumonia. Five months after infection, all patients still had neutralizing antibodies, but the geometric mean titer decreased significantly (219.4 at 2 months vs. 143.7 at 5 months; $p=0.03$ ). In the linear regression model, the decline was significantly associated with the antibody levels at 2 months as measured by ELISA $(r=0.536, p=0.02)$ and the neutralization assay $(r=0.563, p=0.02)$ (Appendix Figure). The waning neutralizing antibody response occurred in $2(40 \%)$ of 5 patients with apparent pneumonia and $2(33 \%)$ of 6 with subtle pneumonia, but none of the asymptomatic persons (Figure).

Determining the longevity of humoral immunity to SARS-CoV-2 is essential to predicting herd immunity to coronavirus disease. Among patients with severe acute respiratory syndrome coronavirus, which is closely related to SARS-CoV-2, a total of $90 \%$ maintained IgG for 2 years and $50 \%$ for 3 years (7). However, humoral immunity to common human coronavirus is short-lived; antibodies against seasonal coronaviruses return to baseline levels by 52 weeks after infection, enabling homologous reinfections (8). A recent study showed that the antibody titers of patients with mild coronavirus disease declined more quickly than did those of patients with severe acute respiratory syndrome (9). 

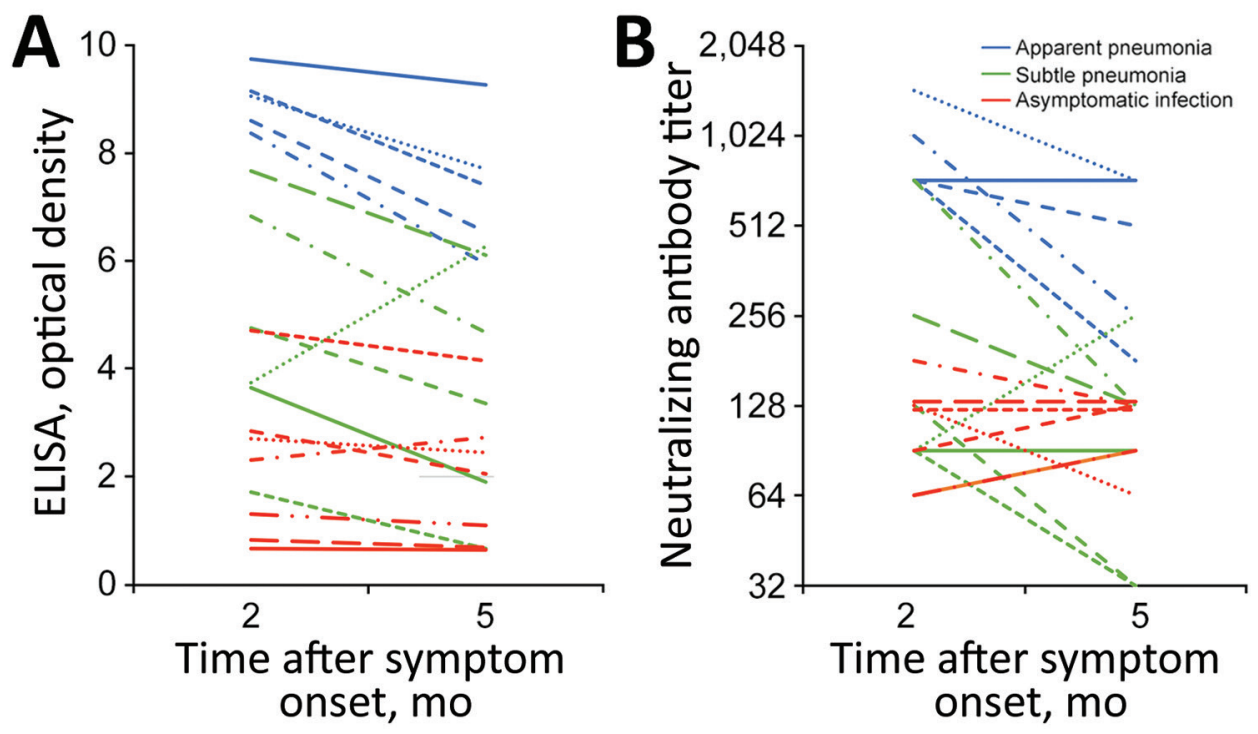

Figure. Waning antibody response against severe acute respiratory virus coronavirus 2, South Korea, 2020. Responses measured by A) ELISA optical density measurements $(p=0.01) ; B)$ neutralizing antibody titers $(p=$ 0.03). Each line indicates data from a single patient.

Our findings demonstrate waning humoral immunity in patients with SARS-CoV-2 infection. We documented the decline of neutralizing antibody titers in asymptomatic and symptomatic patients. In this study, the initial neutralizing antibody reaction appeared to correlate with the severity of the disease. However, patients with pneumonia were considerably older than asymptomatic persons, and increasing age is associated with a stronger neutralizing antibody response (10). In this study, neutralizing antibody titer decreased more in symptomatic than asymptomatic patients. Our study reinforces the concern that naturally acquired humoral immunity against SARS-CoV-2 might not be long-lasting.

\section{Acknowledgments}

We thank Kyung Sook Ahn for administrative support. We thank Areum Jo and Su Jin Choi for technical support.

This project was supported by the research fund of Seoul National University Hospital (grant no. 04-2020-0030). The funding agencies had no role in the design and conduct of the study; collection, management, analysis, and interpretation of the data; preparation, review, or approval of the manuscript; and decision to submit the manuscript for publication.

\section{About the Author}

Dr. Choe is a clinical scientist at Seoul National University Hospital. His research interests focus on preventing healthcare-associated infection and responding to emerging infectious diseases.

\section{References}

1. Choe PG, Kang CK, Suh HJ, Jung J, Kang E, Lee SY, et al. Antibody responses to SARS-CoV-2 at 8 weeks postinfection in asymptomatic patients. Emerg Infect Dis. 2020;26:2484-7. https://doi.org/10.3201/eid2610.202211

2. Long QX, Tang XJ, Shi QL, Li Q, Deng HJ, Yuan J, et al. Clinical and immunological assessment of asymptomatic SARS-CoV-2 infections. Nat Med. 2020;26:1200-4. https:/ / doi.org/10.1038/s41591-020-0965-6

3. Kang E, Lee SY, Jung H, Kim MS, Cho B, Kim YS. Operating protocols of a community treatment center for isolation of patients with coronavirus disease, South Korea. Emerg Infect Dis. 2020;26:2329-37. https:/ / doi.org/10.3201/ eid2610.201460

4. Choe PG, Kang EK, Lee SY, Oh B, Im D, Lee HY, et al. Selecting coronavirus disease 2019 patients with negligible risk of progression: early experience from non-hospital isolation facility in Korea. Korean J Intern Med. 2020;35:765-70. https:/ / doi.org/10.3904/ kjim.2020.159

5. Shen C, Wang Z, Zhao F, Yang Y, Li J, Yuan J, et al. Treatment of 5 critically ill patients with COVID-19 with convalescent plasma. JAMA. 2020;323:1582-9. https://doi.org/10.1001/jama.2020.4783

6. Park WB, Kwon NJ, Choi SJ, Kang CK, Choe PG, Kim JY, et al. Virus isolation from the first patient with SARS-CoV-2 in Korea. J Korean Med Sci. 2020;35:e84. https:// doi.org/10.3346/jkms.2020.35.e84

7. Wu LP, Wang NC, Chang YH, Tian XY, Na DY, Zhang LY, et al. Duration of antibody responses after severe acute respiratory syndrome. Emerg Infect Dis. 2007;13:1562-4. https://doi.org/10.3201/eid1310.070576

8. Callow KA, Parry HF, Sergeant M, Tyrrell DA.

The time course of the immune response to experimental coronavirus infection of man. Epidemiol Infect. 1990;105:435-46. https:/ / doi.org/10.1017/ S0950268800048019

9. Ibarrondo FJ, Fulcher JA, Goodman-Meza D, Elliott J, Hofmann C, Hausner MA, et al. Rapid decay of anti-SARS-CoV-2 antibodies in persons with mild Covid-19. N Engl J Med. 2020;383:1085-7. https://doi.org/10.1056/ NEJMc2025179 
10. Wang X, Guo X, Xin Q, Pan Y, Hu Y, Li J, et al. Neutralizing antibodies responses to SARS-CoV-2 in COVID-19 inpatients and convalescent patients. Clin Infect Dis. 2020;ciaa721. https://doi.org/10.1093/cid/ciaa721

Address for correspondence: Wan Beom Park or Myoung-don Oh, Department of Internal Medicine, Seoul National University College of Medicine, 103 Daehak-ro, Jongno-gu, Seoul 03080, South Korea; email: wbpakr1@snu.ac.kr or mdohmd@snu.ac.kr

\section{Postmortem Stability of SARS-CoV-2 in Nasopharyngeal Mucosa}

\begin{abstract}
Fabian Heinrich, Kira Meißner, Felicia Langenwalder, Klaus Püschel, Dominik Nörz, Armin Hoffmann, Marc Lütgehetmann, Martin Aepfelbacher, Eric Bibiza-

Freiwald, Susanne Pfefferle, Axel Heinemann
\end{abstract}

Author affiliation: University Medical Center Hamburg-Eppendorf, Hamburg, Germany

DOI: https://doi.org/10.3201/eid2701.203112

Analyses of infection chains have demonstrated that severe acute respiratory syndrome coronavirus 2 is highly transmissive. However, data on postmortem stability and infectivity are lacking. Our finding of nasopharyngeal viral RNA stability in 79 corpses showed no time-dependent decrease. Maintained infectivity is supported by virus isolation up to 35 hours postmortem.

$\mathrm{D}$ etailed analyses of severe acute respiratory syndrome coronavirus 2 (SARS-CoV-2) transmission have shown the virus to be highly transmissible through droplet and contact-transmitted viral spreading; reproduction indices were 2.2-3.6 (1). Amid the coronavirus disease (COVID-19) pandemic, case-fatality rates of up to $9.26 \%$ occur in areas hard-struck by SARS-CoV-2 (2). The likelihood of virus transmission through deceased persons remains unclear. However, in recent pandemics of influenza, high and sustainable virus stability and infectivity within corpses were demonstrated $(3,4)$, necessitating careful and conscious handling. To determine the possibility of SARS-CoV-2 transmission through deceased persons, we conducted a study of postmortem viral RNA stability.
The federal state of Hamburg, Germany, has mandated autopsies since March 2020 in accordance with the German Infection Protection Act for all patients with reverse transcription PCR (RT-PCR)-confirmed SARS-CoV-2 infection. Data and sample acquisition for the study were performed during March 22-May 1,2020 . To confirm the initial diagnosis and quantify the viral load in the corpses, nasopharyngeal swab samples (ESwab; Copan, https://products.copangroup.com) were taken at patient admission to the Department of Legal Medicine (University Medical Center Hamburg-Eppendorf). Corpses were stored at $4^{\circ} \mathrm{C}$ in the refrigerator. Antemortem and postmortem nasopharyngeal swab samples were taken according to recent standards (5) by trained, medically qualified personnel to ensure maximum reliability and consistent quality. Samples were analyzed for SARS-CoV-2 RNA as described previously (6).

The Ethics Committee of the Hamburg Chamber of Physicians approved the study (no. PV7311). The local clinical institutional review board, complying with the Declaration of Helsinki, also approved the study.

Antemortem nasopharyngeal swab samples (Appendix Figure, https://wwwnc.cdc.gov/EID/ article/27/1/20-3112-App1.pdf) were collected by medical staff at the intensive care unit of the University Medical Center Hamburg and by general practitioners from on-call duty at a median of 6 days (range 2-14 [interquartile range (IQR) 6.3]) before death $(n=10)$. Using a Wilcoxon test for paired data, we did not detect any effect of the event of death on the SARS-CoV-2 RNA load ( $=-5 ; p=0.85)$. We found no correlation between the postmortem interval (time of death until cooling at $4^{\circ} \mathrm{C}$; median 17.8 [range 2.7482.6]) hours and the viral RNA loads of corpses, as indicated by Spearman correlation of 79 matched datasets (Figure, panel A).

To analyze postmortem stability of SARS-CoV-2 RNA, we selected 11 corpses with short postmortem intervals for a detailed observation over 7 days (168 hours) (Table). The median postmortem interval was 5.7 (range 2.9-32.0 [IQR 6.9]) hours. The median cycle threshold $\left(\mathrm{C}_{\mathrm{t}}\right)$ of SARS-CoV-2 RNA in swab samples taken at admission was 29.52 (range 15.250.0 [IQR 22.5]) (Figure, panel A). We determined viral load in a series of 9 sequential pharyngeal swab samples (time points $0,12,24,36,48,60$, 72, 96, and 168 hours after admission). We consistently detected SARS-CoV-2 RNA at constant levels at all time points analyzed (Figure, panel B), except for patient 7 at 0,12 , and 24 hours after admission and patient 8 at admission. Because subsequent samples were positive for all corpses, we attributed those 